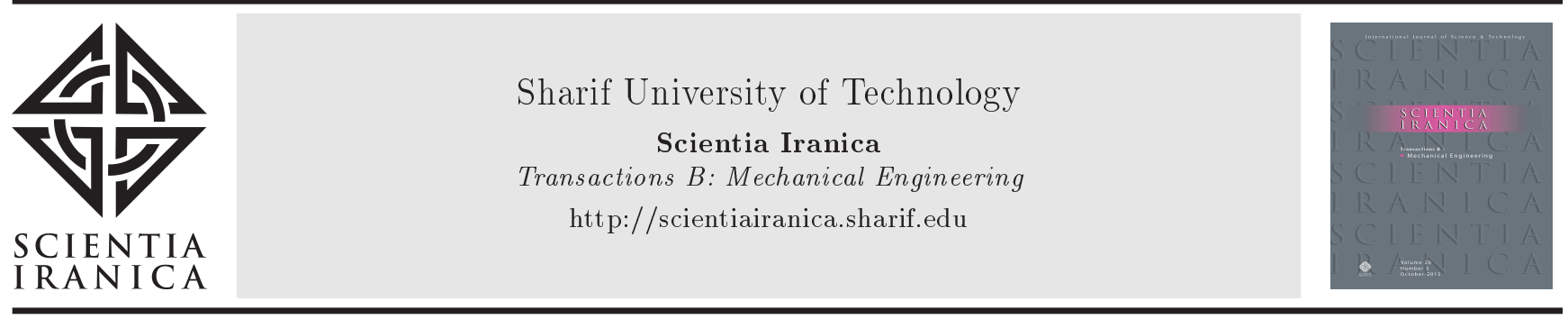

\title{
Optimization of multiple transmission layouts for minimal energy consumption of a battery electric vehicle
}

\author{
T. Farjam, M. Saadat Foumani*, and M. Delkhosh \\ School of Mechanical Engineering, Sharif University of Technology, Tehran, P.O. Box 11155-9567, Iran.
}

Received 23 July 2017; received in revised form 10 October 2017; accepted 21 July 2018

\author{
KEYWORDS \\ Battery electric \\ vehicle; \\ Continuously variable \\ transmission; \\ Power-split \\ transmission; \\ Energy consumption; \\ Particle swarm \\ optimization.
}

\begin{abstract}
Battery Electric Vehicles (BEVs) are a promising solution for reducing the impacts of passenger vehicles on the environment. However, their driving range is restricted due to the limitations of battery technologies. This range can be extended by adoption of multiple-speed transmissions. Most of the comparisons in the related studies are based on non-optimal designs or limited to modal driving cycles. Furthermore, the impact of PowerSplit Continuously Variable Transmission (PS-CVT) layout with type III power flow on the power consumption of BEVs has never been examined. In this paper, single-, two-, and three-speed transmissions along with PS-CVTs with type I and III power flows are optimized for a case study of BEV. Furthermore, the effects of push belt and full-toroidal CVTs in construction of PS-CVT are compared. The results demonstrated that a PS-CVT with type I power flow equipped with the full-toroidal CVT had the best performance. However, it reduced the energy consumption by $0.36 \%$ compared to the optimal two-speed layout. In addition, its ratio range was more limited, which could negatively impact the dynamic performance. Finally, simulation of the optimal designs along a different cycle proved that the obtained results were consistent, regardless of the driving cycle.

(C) 2019 Sharif University of Technology. All rights reserved.
\end{abstract}

\section{Introduction}

Internal Combustion Engines (ICEs) are the dominant power source for vehicles [1]. However, due to the adverse effects of their emissions, manufacturers have to comply with strict regulations regarding air pollution [2]. Despite all the innovations and improvements regarding ICEs, emissions are an inherent part of them. Therefore, many manufacturers have shifted their attention to Electric Motors (EMs) as a possible

*. Corresponding author. Tel.: +982166165534

E-mail addresses: farjam_t@mech.sharif.ir (T. Farjam);

m_saadat@sharif.ir (M. Saadat Foumani);

m_delkhosh@mech.sharif.edu (M. Delkhosh).

doi: $10.24200 /$ sci.2018.20783 replacement. This has led to the emergence of Hybrid Electric Vehicles (HEVs) and Battery Electric Vehicles (BEVs).

Unlike HEVs, BEVs do not contain any ICEs and thus, are considered to have zero emission. In BEVs, the required energy for the operation of EM is supplied by a battery pack, which is often referred to as the Energy Storage System (ESS). Despite its considerable weight, ESS can store a relatively low amount of energy due to its low energy density [3]. Since increasing the weight can negatively impact the performance and energy consumption of BEVs, a major challenge faced by $\mathrm{BEV}$ manufacturers is how to increase its range without increasing the weight of ESS. Hence, other possible solutions that result in higher overall efficiency and thus, less energy consumption are more beneficial.

In addition to zero emission, EMs can produce 
high torques at low speeds, which is a favorable characteristic for the power source of a vehicle. Furthermore, they can operate at relatively higher speeds and continuously produce maximum power. Hence, adoption of a single-speed transmission in BEVs can result in adequate dynamic performance (i.e., acceleration, gradeability, and top speed) [4]. However, the efficiency of EM is not constant and depends on its operating conditions. Consequently, coupling a multispeed transmission with EM can ensure better EM efficiencies. This improved efficiency can lead to lower energy consumption and thus, a higher range for BEV without increasing the weight of ESS [5].

Many researchers have studied the effects of using a two-speed transmission in BEVs [4-8]. They all have delivered consistent results showing that this configuration results in low energy consumption compared with a single-speed transmission. Furthermore, study of a three-speed transmission has shown similar results. Zhang et al. [9] optimized a three-speed transmission for a BEV and reported its 9.3\% less energy consumption than that of the original singlespeed transmission.

Another type of transmission, which has been widely studied in conventional vehicles is Continuously Variable Transmission (CVT) [10]. CVTs are capable of providing a continuous ratio change. Therefore, optimum operating condition of the power source can be ensured. However, their adoption in BEV has rarely been discussed and the results are inconsistent. Sorniotti et al. [11] studied the effect of single- and two-speed transmissions along with CVT on 4 case study vehicles with the assumption that no loss would occur in CVT. Although the results were slightly in favor of CVT, they argued that due to complex design and losses in real situations, the two-speed configuration was superior. On the other hand, Ruan et al. [12] showed that the energy consumption of a BEV equipped with CVT in Urban Dynamometer Driving Schedule (UDDS) was substantially less than that of a similar case with a two-speed transmission and thus, its application was justified.

The main drawbacks of CVT are its limited ratio range, low efficiency, and restricted power transfer capacity [13]. In order to resolve these weaknesses, a Fixed Ratio (FR) mechanism and Planetary Gear (PG) train can be used in conjunction with CVT. This type of transmission is known as Power-Split Continuously Variable Transmission (PS-CVT) and can perform better than the single CVT in certain operating conditions [14]. Bottiglione et al. [15] studied this type of transmission for a $\mathrm{BEV}$. The results indicated that a type I PS-CVT would have lower energy consumption than single- and two-speed transmissions, full-toroidal and half-toroidal CVTs, and type II PS-CVT.

One major flaw in the studied literature is that the gear ratios of the compared layouts have not been optimized for minimum energy consumption $[6,12,15$ 22]. Thus, the results are inconclusive. Furthermore, most simulations have been conducted along modal driving cycles $[6,9,12,15,16,19]$. Therefore, the results may not apply to real driving situations. The aim of this paper is to minimize the energy consumption of a BEV employing different types of transmissions along a transient driving cycle. The main focus will be on modeling and optimization of type I and type III PS-CVTs. Moreover, two types of CVT, namely fulltoroidal and push belt, will be used in these structures to study the effects of their unique characteristics on the final results. In addition to PS-CVTs, single-, two, and three-speed transmissions will be studied. Not only the ratios, but also the shifting pattern will be optimized. Finally, the BEV containing the optimum design of each layout will be simulated along a different cycle to study the consistency of results.

\section{BEV model}

The studied vehicle is the result of conversion of a conventional passenger vehicle [23]. The key parameters of its components, which have been used in the modeling process, are summarized in Table 1. Furthermore, characteristics of the main components will be briefly discussed. Due to the relatively complex structure, modeling of PS-CVT will be discussed in detail in the next section.

\subsection{EM and ESS}

The studied BEV is equipped with a permanent magnet EM unit. This unit is capable of producing 85 $\mathrm{kW}$ (peak) power and $255 \mathrm{Nm}$ (peak) torque, and is able to operate in constant power above its base speed (3183 rpm). This EM can operate at speeds up to 10250 rpm and its efficiency is higher than $80 \%$ in most of the operating regions as depicted in Figure 1.

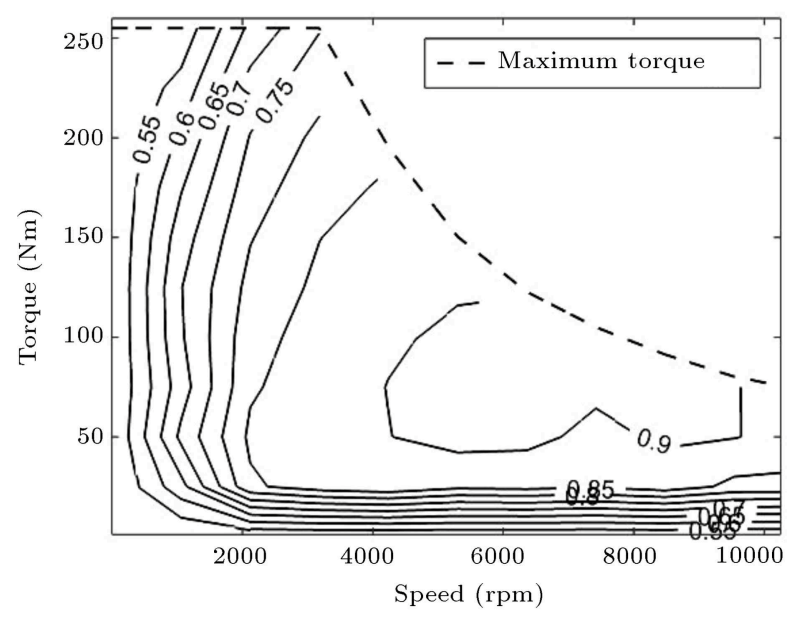

Figure 1. Efficiency contours of the EM. 
Table 1. Vehicle parameters.

\begin{tabular}{lc}
\hline Parameter & Value \\
\hline Vehicle: & \\
Coast-down coefficient $\left(f_{0}\right)$ & $123.41 \mathrm{~N}$ \\
Coast-down coefficient $\left(f_{1}\right)$ & $2.873 \mathrm{Ns} / \mathrm{m}$ \\
Coast-down coefficient $\left(f_{2}\right)$ & $0.311 \mathrm{Ns}^{2} / \mathrm{m}^{2}$ \\
Tire radius & $0.301 \mathrm{~m}$ \\
Curb weight & $1304 \mathrm{~kg}$ \\
Transmission: & \\
Gearbox ratio & 0.488 \\
Gearbox efficiency & $98 \%$ \\
Final drive ratio & 0.256 \\
Final drive efficiency & $97 \%$ \\
EM: & \\
Maximum power & $85 \mathrm{~kW}$ \\
Continuous power & $50 \mathrm{~kW}$ \\
Maximum torque & $255 \mathrm{Nm}$ \\
Continuous torque & $95 \mathrm{Nm}$ \\
Operating range & $0-10250 \mathrm{rpm}$ \\
ESS: & \\
Number of modules & 192 \\
Energy content & $28 \mathrm{kWh}$ \\
Nominal voltage & $350 \mathrm{~V}$ \\
\hline
\end{tabular}

The ESS can store $28 \mathrm{kWh}$ energy and generate the $350 \mathrm{~V}$ nominal voltage required by the EM. ESS forms an extremely complex time-varying and nonlinear system. In this research, ESS is modeled according to the internal resistance model similar to ADVISOR. The thermal behavior of ESS and the effect of temperature variation on its characteristics have been ignored for the sake of simplicity. Charging behavior has not been taken into consideration since the effect of regenerative braking is negligible [4]. The internal discharge resistance of ESS modules varies with their state of charge as shown in Figure 2.

\subsection{Transmission}

The most common type of transmission adopted in BEVs is single-speed. The designed ratio should satisfy gradeability, acceleration performance, and top speed constraints, simultaneously. While the first two call for a low speed ratio, the latter requires higher ratios; thus, the final design is a trade-off. The studied vehicle is fitted with a single-speed gearbox with the ratio and efficiency reported in Table 1 .

Two-speed transmissions are capable of resulting in better overall performance of BEV. In this case, the first gear is designed to achieve the desired gradeablity and acceleration performance, while the second gear ensures that the specified top speed is achievable.

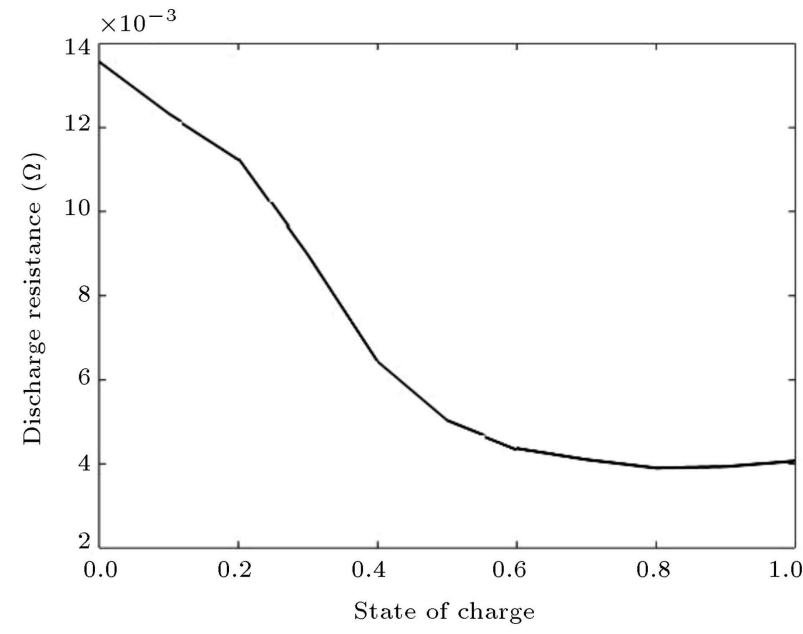

Figure 2. Internal discharge resistance of ESS modules as a function of state of charge.

Hence, these constraints can be satisfied separately, thus providing more feasible designs. Additionally, with the adoption of an optimized shifting pattern, EM can operate in more optimum regions. Therefore, lower energy consumption can be achieved. The design of the first and last gear ratios in a three-speed transmission is similar to that in the two-speed case. However, with the addition of a middle gear, more optimal operating conditions can be provided for EM.

The downside of adding gear sets is higher losses in the transmission system. Single-speed transmission has the least number of moving parts and a typical efficiency of $98 \%$. Two-speed and three-speed transmissions have more rotating parts and thus, lower efficiency of $95 \%$ and $92 \%$, respectively [20]. The casestudy vehicle includes a final drive mechanism in which ratio and efficiency are 0.256 and $97 \%$, respectively.

\subsection{Chassis}

The BEV is considered as a lumped mass moving only along the longitudinal axis. The elementary equation governing the movement of the vehicle has the following form:

$$
m \frac{d v(t)}{d t}=F_{t}-F_{r e s}=F_{t}-\left[f_{0}+f_{1} v+f_{2} v^{2}\right],
$$

where $m$ is dynamic mass of the vehicle, $v$ is its velocity, $F_{t}$ is the traction force on wheels, and $F_{\text {res }}$ is the resistance force (sum of rolling resistance and aerodynamic drag). The resistance force can be derived more accurately by using the results from coast-down test. Hence, constants $f_{0}, f_{1}$, and $f_{2}$ are implemented in this study and their respective values are reported in Table 1 .

\section{PS-CVT architectures}

As stated before, PS-CVT is comprised of a PG, FR, and CVT in the transmission system. This type 
of transmission can offer a wider speed ratio range, higher power transmission capacity, and better overall efficiency than the CVT (in certain operating conditions). PS-CVT can be categorized as series or parallel depending on the arrangement of its components. In a series arrangement, CVT and PG are directly connected to the input shaft (Figure 3(a)), while parallel PS-CVT is constructed by direct connection of CVT and FR to the input shaft (Figure $3(\mathrm{~b})$ ). The CVTs in this study cannot endure speeds higher than $6000 \mathrm{rpm}$; thus, a single reduction gear $\left(\mathrm{FR}_{\mathrm{in}}\right)$ must be embedded in the input. This additional component ensures that the EM can operate even at its peak speed without harming the CVT.

As depicted in Figure 4, three types of power flows are possible in PS-CVT, which are determined by the design of FR and PG. As mentioned in Ref. [24], with a type II power flow, the input power of CVT will always be larger than that of PS-CVT. Since CVT has the least efficiency among all PS-CVT components, the more the power transferred through the CVT, the less the efficiency of PS-CVT will be. Furthermore, the power transfer capacity will be more restricted. In contrast, with a type I power flow, the input power to the CVT is smaller than the total input power to the PS-CVT in some ranges, which leads to higher efficiency in those ranges.

Mangialardi and Mantriota [14] derived the necessary equations to calculate the efficiency of type I and II flows. Their study showed that series configuration was more beneficial and type I flow would lead to better results in terms of efficiency. However, further study of type III flow was disregarded due to the specific conditions under which it occurred.

Unlike the first two types of power flows, the type III flow will not result in power recirculation, thus better efficiency characteristics are expected. This attribute makes this type of flow worthy of comparison to type I in terms of efficiency. However, it has been shown that this type of flow occurs only when the ratio range of $\mathrm{PS}-\mathrm{CVT}$ is smaller than that of CVT [25]. Hence, its study has largely been neglected $[14,15,26,27]$. Nevertheless, as the results will indicate, the optimal designs satisfy this condition. This type of flow has been investigated only in some special configurations $[13,25,28,29]$ designed to have a range equal to CVT. Therefore, in order to investigate the characteristics of the type III power flow in a series arrangement with a limited range and calculate its efficiency, kinematic analysis should be conducted.

The transmission ratios of each component can be determined using the following equations, where $\omega_{i}$ denotes the rotational speed of the $i$ th path:

$$
\begin{aligned}
& \tau_{c v t}=\frac{\omega_{5}}{\omega_{3}}, \quad \tau_{F R}=\frac{\omega_{6}}{\omega_{5}}, \quad \tau_{P G}=\frac{\omega_{7}-\omega_{6}}{\omega_{4}-\omega_{6}}, \\
& \omega_{2}=\omega_{3}=\omega_{4} .
\end{aligned}
$$

Applying the power conservation equation to PG results in Eq. (3), which can be rewritten as Eq. (4):

$$
\begin{aligned}
& \eta_{P G} p_{4}+\eta_{P G} p_{6}+p_{7}=0, \\
& \eta_{P G}\left(\left|T_{4} \omega_{4}\right|+\left|T_{6} \omega_{6}\right|\right)-\left|T_{7} \omega_{7}\right|=0,
\end{aligned}
$$

where $\eta_{P G}$ denotes the efficiency of PG. Assuming that $\tau_{P S-C V T}$ and $\tau_{C V T}$ only take positive values, Eq. (5) can be derived in which $k_{1}$ and $k_{2}$, accordingly, have positive values. Hence, increasing $\tau_{C V T}$ results in the

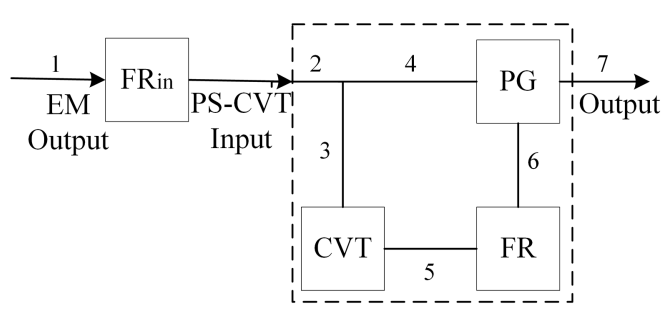

(a)

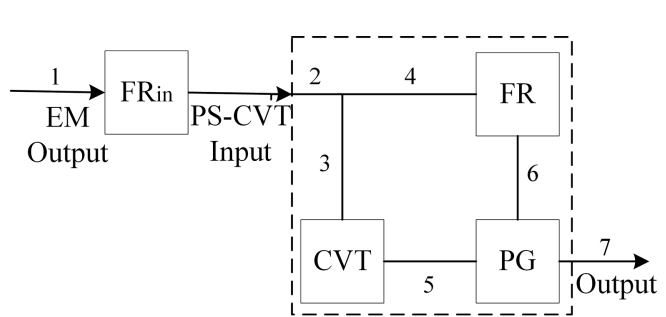

(b)

Figure 3. PS-CVT arrangements: (a) Series and (b) parallel.
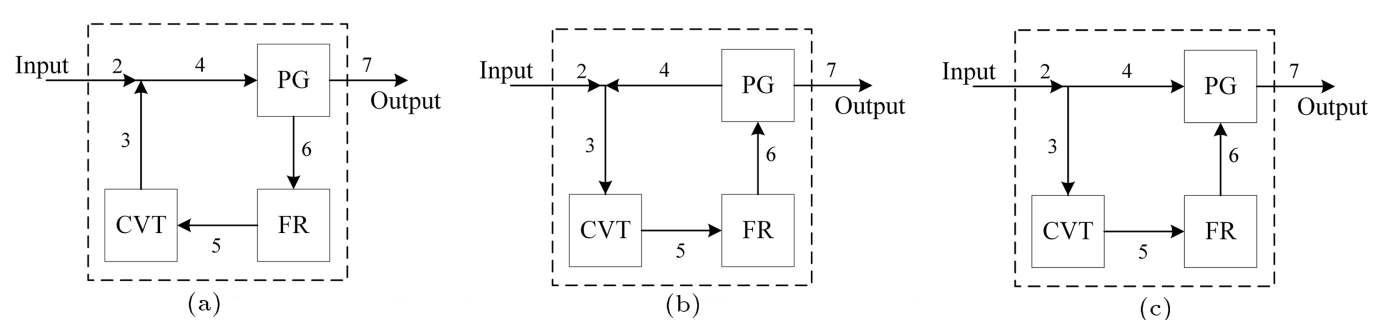

Figure 4. Power flows in a series PS-CVT: (a) Type I, (b) type II, and (c) type III. 
increase in total transmission ratio:

$$
\begin{aligned}
\tau_{P S-C V T} & =\eta_{P G}\left(\left|\frac{T_{4}}{T_{7}}+\right| \frac{T_{6}}{T_{7}}|| \tau_{F R} \tau_{C V T} \mid\right) \\
& =k_{1}+k_{2} \tau_{C V T} .
\end{aligned}
$$

Using Eq. (1) and the relation derived in Eq. (5), the ratios of $\mathrm{PG}$ and $\mathrm{FR}$ can be derived as follows:

$$
\begin{aligned}
\tau_{P G} & =\frac{\tau_{C V T_{\max }} \tau_{I V T_{\min }}-\tau_{C V T_{\min }} \tau_{I V T_{\max }}}{\tau_{C V T_{\max }}-\tau_{C V T_{\min }}}, \\
\tau_{F R} & =\frac{\tau_{I V T_{\max }}-\tau_{P G}}{\left.\tau_{C V T_{\max }\left(1-\tau_{P G}\right)}\right)}
\end{aligned}
$$

which shows that by determining the desired range for the PS-CVT, the design parameters of other components can be derived.

Following a similar approach to that in [25], the necessary condition for occurrence is obtained as follows:

$$
\frac{\tau_{P S-C V T_{\max }}}{\tau_{P S-C V T_{\min }}} \leq \frac{\tau_{C V T_{\max }}}{\tau_{C V T_{\min }}} .
$$

Finally, the efficiency of a series PS-CVT with a type III power flow will be:

$\eta_{P S-C V T}$

$$
=\frac{\eta_{F R} \eta_{P G} \eta_{C V T}\left(\frac{1}{\tau_{C V T} \tau_{F R}}-1\right) \tau_{P S-C V T}}{\eta_{P G}\left(1-\eta_{F R} \eta_{C V T}\right)+\tau_{P S-C V T}\left(\frac{\eta_{F R} \eta_{C V T}}{\tau_{C V T} \tau_{F R}}-1\right)} .(9)
$$

As it can be seen, the overall efficiency of PS-CVT is a function of the efficiency of its components. The losses in FR can be assumed constant [14]; thus, its efficiency is set to $98 \%$. However, PG and CVT have more complex behaviors in terms of loss. Assuming that the losses in PG are only the result of friction between gear train teeth, its efficiency can be calculated according to [30]. CVT has the least efficiency among all these components. Therefore, accurate modeling of its losses is essential.

The most common types of CVT employed in automotive industry are toroidal and push belt, and both can be adopted in the construction of PS-CVT. Although both types offer a continuous change of speed ratio, their efficiency depends highly on their working conditions. In this research, a commercial prototype of both types will be modeled and studied. The studied push belt type CVT is a commercial product and can transfer torques up to $225 \mathrm{Nm}$. Moreover, it is capable of providing ratios between 0.41 and 2.33, continuously. Rather than application of theoretical equations, results of real-life operation are used for estimation of its efficiency. The efficiency at any desired state can be estimated by interpolation between available data points. Figure 5 shows the variation of efficiency, as a function of input torque, and speed while the CVT is working at its minimum speed ratio.

The efficiency of the selected full-toroidal CVT can be calculated analytically using the model presented in [31] in which the efficiency is considered as a function of geometry, condition of the lubricating oil, and operating conditions such as speed ratio, input torque, and rotational speed. The efficiency characteristics of this type are considerably different from those of the push belt CVT. As shown in Figure 6 for $\tau_{C V T}=1$, a push belt CVT has higher efficiency while operating at higher torques; conversely, a fulltoroidal CVT operates better at lower torques. Hence, the type of CVT used in the construction of PSCVT can affect its overall efficiency significantly along a driving cycle. Therefore, the efficiency model of both types should be employed to calculate the overall performance of powertrain.

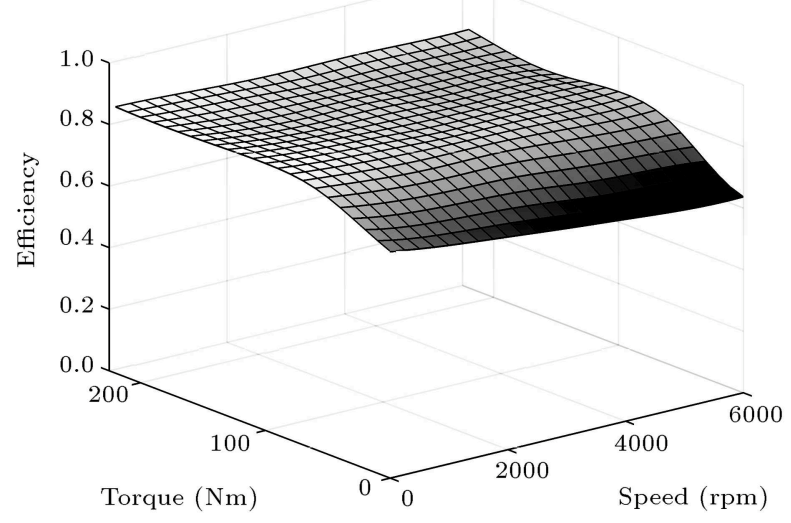

Figure 5. Efficiency of push belt CVT as a function of its input torque and speed while working at minimum speed ratio.

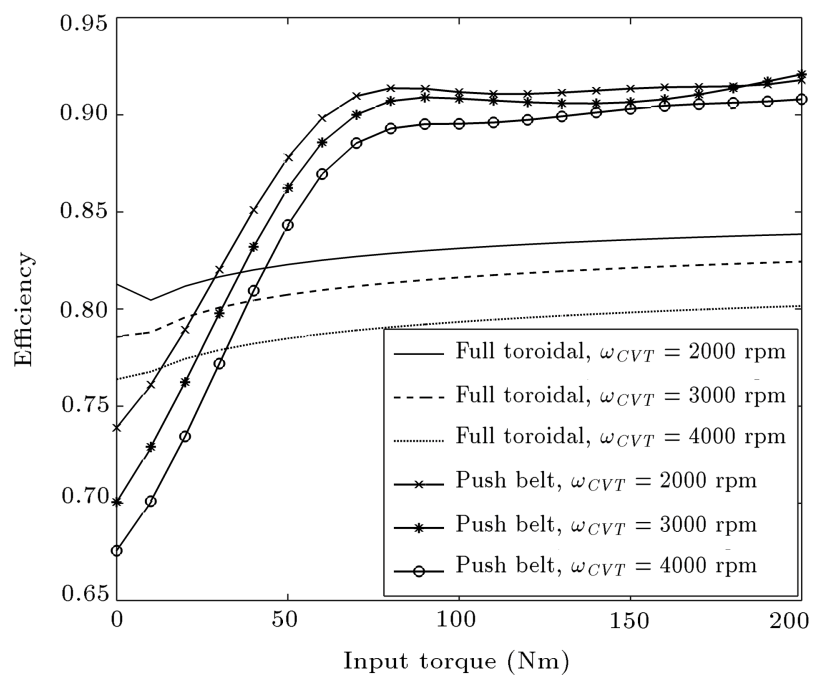

Figure 6. Efficiency characteristics of push belt and full-toroidal CVTs at $\tau_{P S-C V T}=1$. 
Table 2. Characteristics of the modeled CVTs.

\begin{tabular}{lcc}
\hline \multicolumn{1}{c}{ Parameter } & Push belt & Full-toroidal \\
\hline Torque capacity & $225 \mathrm{Nm}$ & $260 \mathrm{Nm}$ \\
Maximum input speed & $6000 \mathrm{rpm}$ & $6000 \mathrm{rpm}$ \\
Peak efficiency & $92 \%$ & $93 \%$ \\
Minimum ratio & 0.41 & 0.77 \\
Maximum ratio & 2.33 & 3.44 \\
\hline
\end{tabular}

Table 2 sums up the key features of these CVTs. As it can be seen, the maximum input speed for both cases is limited to $6000 \mathrm{rpm}$.

\section{Optimization method}

The main target of this paper is to minimize the energy consumption of the BEV along a transient driving cycle. The consumed energy mainly depends on the performance of the EM and transmission. In a BEV equipped with a single-speed transmission, EM can operate at a certain operating point depending on the torque and speed required at wheel. Therefore, a brute force algorithm can be utilized to find the optimal ratio. However, other proposed configurations offer multiple operating conditions for EM at each time step. Therefore, a suitable optimization algorithm should be employed to find the optimal ratios and gear selection pattern.

For the present study, Particle Swarm Optimization (PSO) algorithm is utilized to optimize the selected layouts. This method has widely been used in various fields because of its high rate of convergence in finding the global minimum [32]. In this method, candidate solutions, which are chosen randomly at the beginning, are treated as particles with specific positions and velocities. At each iteration of the algorithm, the velocities and positions of these particles are updated with respect to their own best position and the overall best position. This procedure continues until the convergence criterion is satisfied. A more detailed description of this algorithm has been presented in [33].

In this optimization, the cost function represents the energy consumption along the driving cycle. The driving cycle in this case is composed of 5 different cycles, namely 15 mode, 10-15 mode, SC03, NYCC, and IM240. Unlike 15 mode and 10-15 mode, which are modal cycles, the rest represent real driving behaviors. Figure 7 represents the speed profile of this cycle. A backward facing simulation is used to determine the value of cost function along this cycle and the final results are verified by the ADVISOR.

Speed and acceleration of the vehicle are known at every time step of this driving cycle. Hence, the torque and speed of the output shaft of transmission can be determined. In case of two- and three-speed

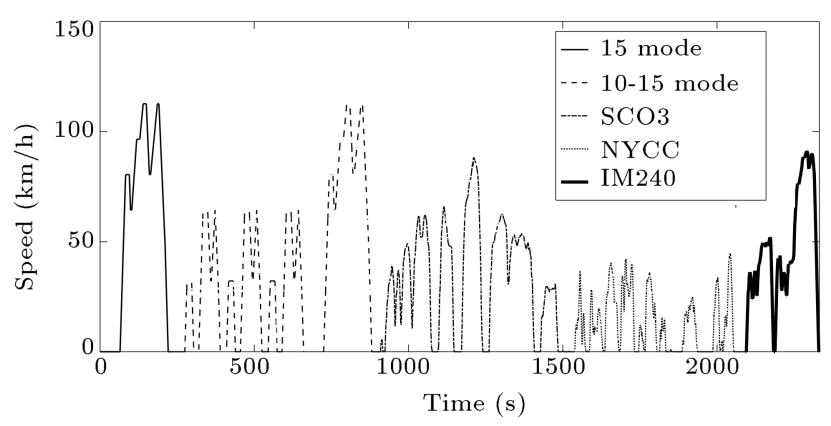

Figure 7. The proposed driving cycle.

transmissions, each gear ratio results in a different operating point for the EM. Therefore, at each step, the ratio which results in the highest EM efficiency is selected.

Unlike the discussed cases, the efficiency of PSCVT varies greatly depending on its ratio, input speed, and torque. Hence, in addition to the operating region of EM, the operating conditions of PS-CVT and thus, its efficiency impact the energy consumption, significantly. Consequently, the overall efficiency of the powertrain must be maximized in order to minimize the energy consumption. This optimization problem can be formulated as follows:

$$
\begin{aligned}
& F_{1}\left(\tau_{P S-C V T}\right) \\
& \quad=\max \left\{\eta_{E M}\left(\tau_{P S-C V T}\right) \cdot \eta_{P S-C V T}\left(\tau_{P S-C V T}\right)\right\}, \\
& F_{2}\left(\tau_{P S-C V T}, F_{1}\right)=\min \{\text { energy consumption }\}, \\
& \tau_{P S-C V T} \epsilon\left[\tau_{P S-C V T_{\min }}, \tau_{P S-C V T_{\max }}\right] .
\end{aligned}
$$

The efficiency of PS-CVT cannot be calculated directly by only knowing its output torque and speed. The procedure carried out to determine its accurate value for every gear ratio uses an initial estimation and determines the required input speed and torque based on this guess. Utilizing the input conditions and speed ratio, the accurate efficiency of PS-CVT is calculated. If this value considerably differs from the initial guess, it will be used as the initial estimation and the procedure is repeated until convergence is attained.

The proposed optimization algorithm for this case follows the flowchart represented in Figure 8. Regarding the required output speed and torque, for every possible ratio, the efficiency of PS-CVT and thus, the input torque and speed can be determined. This determines the operating point of EM. By increasing the speed ratio from $\tau_{P S-C V T_{\min }}$ up to $\tau_{P S-C V T_{\max }}$, the product of the efficiency of EM and PS-CVT is calculated and finally, the ratio corresponding to maximum overall efficiency is selected. It should be noted that the ratios which result in torques and speeds outside the working range of the EM are not acceptable. 


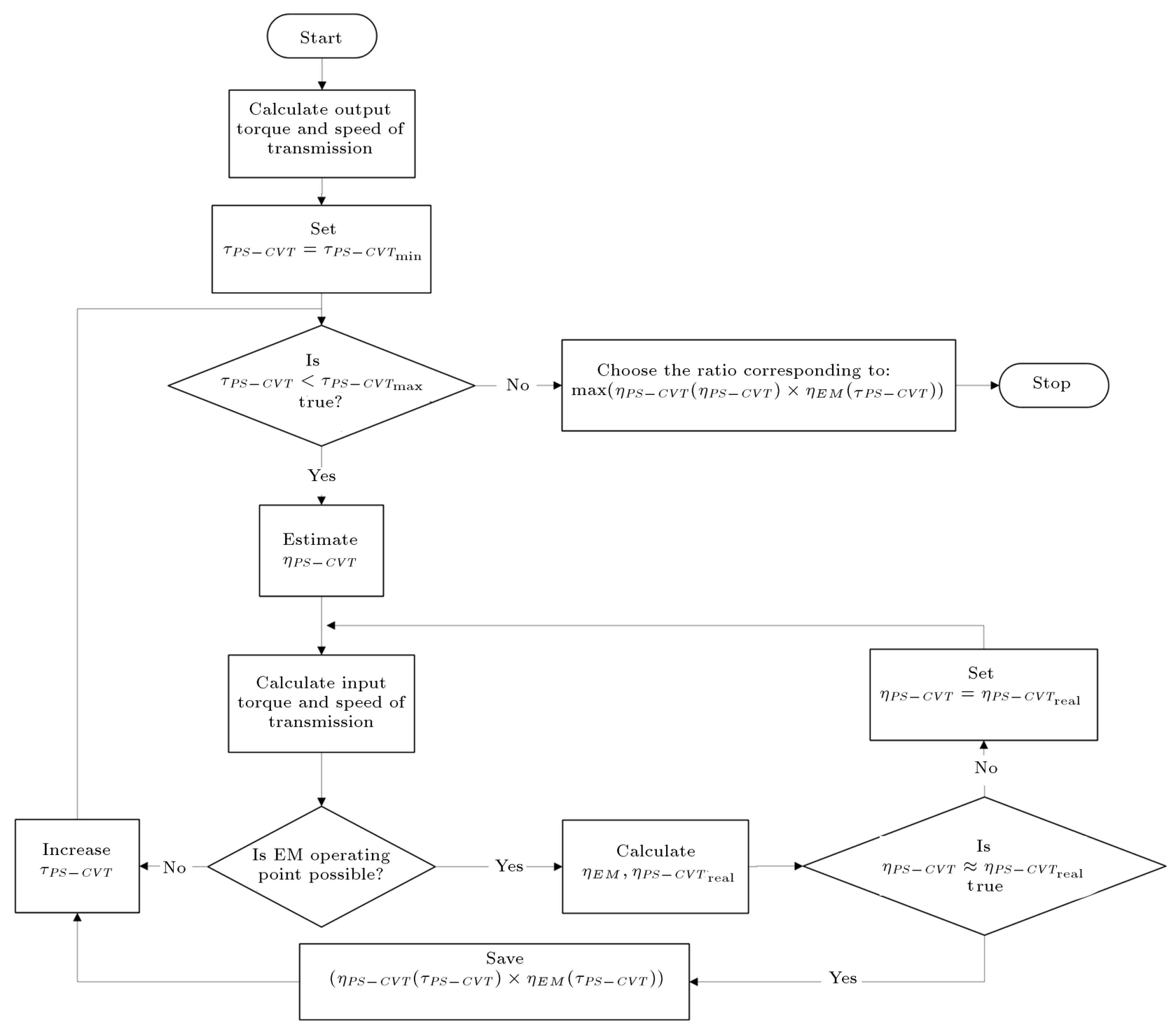

Figure 8. Flowchart of the optimization procedure for PS-CVT.

Similarly, ratios corresponding to input CVT torques larger than its capacity are ignored.

A crucial step in the optimization procedure is establishing the constraints. The constraints mentioned so far are the ones concerned with the maximum capacity of components. However, as mentioned earlier, gradeability and acceleration performance set an upper bound on the possible values of the minimum ratio. Similarly, the maximum ratio corresponds to top speed. Therefore, the desired top speed requires the designed highest ratio to be larger than a specific value. In the present study, these limits are assigned according to PNGV performance constraints [34]. The proposed acceleration performance is more demanding than gradeability. Therefore, an acceleration time less than 12 seconds from 0 to $97 \mathrm{~km} / \mathrm{h}$ bounds the lowest ratio. Furthermore, the designed transmission should enable the BEV to reach speeds higher than 137
Table 3. Bounds on minimum and maximum ratios.

\begin{tabular}{ccc}
\hline Transmission & $\begin{array}{c}\text { Maximum value } \\
\text { for } \boldsymbol{\tau}_{\min }\end{array}$ & $\begin{array}{c}\text { Minimum value } \\
\text { for } \boldsymbol{\tau}_{\max }\end{array}$ \\
\hline Single-speed & 0.92 & 0.46 \\
Two-speed & 0.88 & 0.46 \\
Three-speed & 0.85 & 0.46 \\
PS-CVT & 1.29 & 0.79 \\
\hline
\end{tabular}

$\mathrm{km} / \mathrm{h}$, which sets the lowest bound on the highest ratio. Using $85 \%$ efficiency for PS-CVT while operating at the minimum ratio and the discussed values for other types of transmission, the parameters reported in Table 3 can be calculated.

\section{Results and discussion}

Using the discussed procedure, optimal design values 
Table 4. Optimal ratios for single-, two-, and three-speed transmissions.

\begin{tabular}{lccc}
\hline Transmission & $\begin{array}{c}\text { First } \\
\text { ratio }\end{array}$ & $\begin{array}{c}\text { Second } \\
\text { ratio }\end{array}$ & $\begin{array}{c}\text { Third } \\
\text { ratio }\end{array}$ \\
\hline Single-speed & 0.58 & - & - \\
Two-speed & 0.298 & 0.705 & - \\
Three-speed & 0.283 & 0.588 & 0.923 \\
\hline
\end{tabular}

can be obtained. Table 4 presents the derived optimum ratios for single-, two-, and three-speed transmissions. As the results indicate, the minimum optimal ratios of two- and three-speed layouts are close. However, the maximum ratio in a three-speed transmission is much larger than that in the two-speed. Furthermore, the carried out procedure determines the minimum and maximum ratios of PS-CVT layouts, which result in minimum energy consumption. Hence, using the proposed shifting algorithm along with a design that leads to these ratios corresponds to maximum vehicle range for these layouts. It is notable that by using these values, the optimal speed ratios of $F R$ and $P G$ can also be determined (see Eqs. (6) and (7)) as represented in Table 5. The results show that the optimum ratio range for a PS-CVT equipped with the push belt CVT and type I power flow (PB-I) is equal to that for the case with the full-toroidal CVT (FT-I). Moreover, the results for the designs with type III flow are roughly the same. The optimal shifting pattern and speed ratios are designed to ensure the least possible energy consumption for the BEV. This quantity along the proposed driving cycle is presented in Table 6. As the results indicate, optimum FT-III design will be the most effective layout at extending the driving range of the BEV. This layout results in $1.86 \%$ lower energy consumption than the original single-speed transmission does. It is interesting to note that a two-speed transmission improves the energy consumption more than both PS-CVT layouts equipped with the push belt CVT do. Furthermore, despite its complex design, FT-I lowers the energy consumption by just $0.34 \%$ compared with the two-speed transmission. Therefore, this slight improvement does not justify its application in the BEV. Moreover, according to Tables 4 and 5, including $\tau_{F R_{\text {in }}}$, the two-speed design covers a larger range. Therefore, this design can also be adopted in vehicles that demand better performance while minimizing the energy consumption at the same time.
Table 6 also indicates that more gear ratios will not necessarily mean lower energy consumption. This can be seen for the case of three-speed transmission. This layout offers more optimal operating conditions for EM. It results in $1.23 \%$ higher EM efficiency along the cycle than the two-speed layout does. However, due to the higher losses of this type of transmission, the overall powertrain efficiency is $1.13 \%$ less. Thus, the overall energy consumption is $2.13 \%$ more than that in the two-speed design.

In order to check the consistency of these results, it is necessary to simulate the vehicle with these designs along another driving cycle. In this study, LA-92 cycle has been selected for this purpose. This cycle is also known as the Unified Cycle Driving Schedule (UCDS) and represents a relatively aggressive driving behavior [35].

Similar to Table 6 , the information in Table 7 can be used to compare the performance of each type of transmission. The results are in agreement with the former case, and FT-I results in slightly lower energy consumption $(0.07 \%)$ than the two-speed transmission. Moreover, the three-speed transmission leads to the least favorable results. Although it offers more favorable operating regions for the EM, its inherent lower efficiency outweighs this advantage.

Both simulations indicate that for each type of power flow, the PS-CVT equipped with the fulltoroidal CVT is more advantageous. As stated earlier, the push belt CVT has higher efficiency when the input torque is large. Conversely, the full-toroidal CVT shows better efficiency characteristics under smaller torques. Figure 9 shows the torque crossing each type of CVT for each power flow type in the first 300 seconds of LA-92 cycle. For a type I flow (Figure 9(a)), with the adoption of the optimal shifting pattern, more torque crosses push belt CVT. However, the input torques are relatively small. Therefore, adoption of FT-I is more beneficial and results in $0.5 \%$ less energy consumption. However, PB-III leads to $1.15 \%$ more energy consumption than FT-III. This is in agreement with the results presented in Figure 9(b). As it can be seen, for a type III flow, the torque applied to the CVT is less than that for the case of type I flow. Furthermore, slightly large torques cross full-toroidal CVT compared with the push belt case. Hence, PB-III will lead to less efficiency.

Table 5. Optimal design of PS-CVT layouts.

\begin{tabular}{lcccc}
\hline Transmission & $\boldsymbol{\tau}_{\boldsymbol{P} \boldsymbol{S}-\boldsymbol{C} \boldsymbol{V} \boldsymbol{T}_{\min }}$ & $\boldsymbol{\tau}_{\boldsymbol{P} \boldsymbol{S}-\boldsymbol{C} \boldsymbol{V} \boldsymbol{T}_{\max }}$ & $\boldsymbol{\tau}_{\boldsymbol{F} \boldsymbol{R}}$ & $\boldsymbol{\tau}_{\boldsymbol{P G}}$ \\
\hline PB-I & 0.790 & 0.991 & 3.074 & 1.034 \\
PB-III & 0.980 & 1.183 & 1.657 & 0.936 \\
FT-I & 0.790 & 0.991 & 1.544 & 1.049 \\
FT-III & 1.001 & 1.211 & 1.566 & 0.952 \\
\hline
\end{tabular}


Table 6. Energy consumption along the proposed driving cycle.

\begin{tabular}{|c|c|c|c|c|c|}
\hline Transmission & $\begin{array}{c}\text { Mean } \\
\text { transmission } \\
\text { efficiency }(\%)\end{array}$ & $\begin{array}{c}\text { Mean EM } \\
\text { efficiency }(\%)\end{array}$ & $\begin{array}{c}\text { Mean } \\
\text { powertrain } \\
\text { efficiency }(\%)\end{array}$ & $\begin{array}{c}\text { Energy } \\
\text { consumption } \\
(\mathrm{kWh}) \\
\end{array}$ & $\begin{array}{c}\text { Improvement } \\
(\%)\end{array}$ \\
\hline Single-speed (original) & 95.06 & 73.03 & 67.99 & 4.15 & 0 \\
\hline Single-speed & 95.06 & 73.51 & 69.88 & 4.13 & 0.38 \\
\hline Two-speed & 92.15 & 76.70 & 70.68 & 4.08 & 1.52 \\
\hline Three-speed & 89.24 & 77.93 & 69.55 & 4.17 & -0.61 \\
\hline PB-I & 96.02 & 73.50 & 70.57 & 4.09 & 1.37 \\
\hline PB-III & 95.03 & 73.70 & 70.01 & 4.12 & 0.55 \\
\hline FT-I & 96.05 & 73.45 & 70.88 & 4.07 & 1.86 \\
\hline FT-III & 96.44 & 73.41 & 70.08 & 4.08 & 1.74 \\
\hline
\end{tabular}

Table 7. Energy consumption of the optimal designs along LA-92 driving cycle.

\begin{tabular}{lccccc}
\hline \multicolumn{1}{c}{ Transmission } & $\begin{array}{c}\text { Mean } \\
\text { transmission } \\
\text { efficiency (\%) }\end{array}$ & $\begin{array}{c}\text { Mean EM } \\
\text { efficiency (\%) }\end{array}$ & $\begin{array}{c}\text { Mean } \\
\text { powertrain } \\
\text { efficiency (\%) }\end{array}$ & $\begin{array}{c}\text { Energy } \\
\text { consumption } \\
\text { (kWh) }\end{array}$ & $\begin{array}{c}\text { Improvement } \\
(\%)\end{array}$ \\
\hline Single-speed (original) & 95.06 & 74.40 & 70.72 & 2.9909 & 0 \\
Single-speed & 95.06 & 74.83 & 71.13 & 2.9859 & 1.17 \\
Two-speed & 92.15 & 78.12 & 71.99 & 2.9430 & 1.6 \\
Three-speed & 89.24 & 79.32 & 70.79 & 3.0053 & -0.48 \\
PB-I & 96.01 & 74.82 & 71.83 & 2.9560 & 1.17 \\
PB-III & 94.93 & 75.09 & 71.26 & 2.9805 & 0.35 \\
FT-I & 96.51 & 74.78 & 72.17 & 2.9411 & 1.67 \\
FT-III & 96.45 & 74.73 & 72.08 & 2.9459 & 1.5 \\
\hline
\end{tabular}

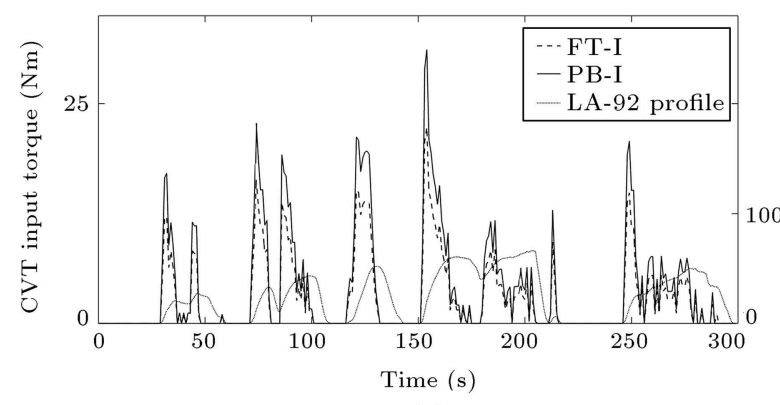

(a)

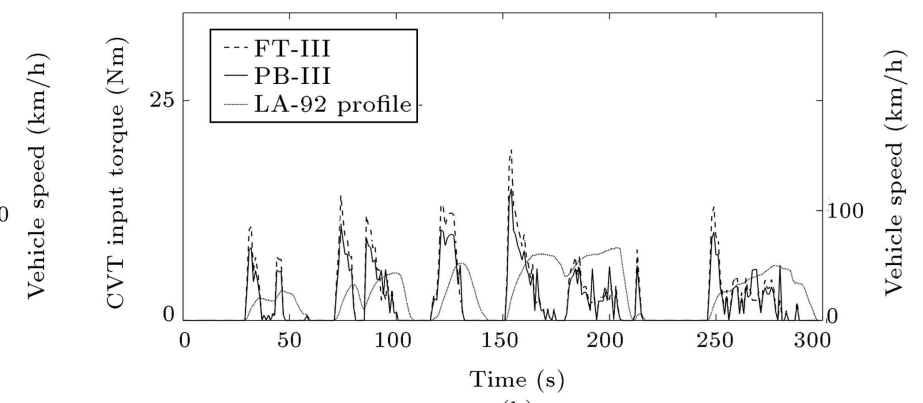

(b)

Figure 9. Torque applied to CVT for a segment of LA-92 cycle: (a) Type I power flow, and (b) type III power flow.

\section{Conclusion}

This paper studied the effect of seven different transmission systems on the energy consumption of a BEV, namely single-, two-, and three-speed PS-CVTs with type I and III power flows equipped with fulltoroidal and push belt CVTs. Using particle swarm optimization, the optimal ratio design of each layout was determined. Furthermore, instead of efficiency of the EM, the efficiency of powertrain was taken into consideration for optimal gear selection.

The results were obtained by conducting simula- tions along a composite driving cycle. This cycle was designed to account for modal and transient driving patterns. The proposed cycle was constructed by putting together 5 different cycles, namely 15 mode, 10-15 mode, SC03, NYCC, and IM240. The results showed that FT-I led to the best results in terms of efficiency. However, these results were not considerably better than those for the optimal two-speed layout. Moreover, regardless of the type of CVT, PS-CVTs with type I power flow had better performance than those with type III. Furthermore, adoption of the fulltoroidal CVT in the construction of PS-CVT produced 
better results than the push belt CVT, regardless of the power flow type.

The BEV with the obtained optimum designs was simulated along LA-92 driving cycle to confirm the consistency of results. The results were in agreement with the former findings. More precisely, FT-I improved the energy consumption by $1.67 \%$ in comparison to the original single-speed transmission. Despite the complex design, this layout offered only $0.07 \%$ improvement compared with the optimal two-speed transmission. Therefore, its application was not justified. Moreover, the ratio range of optimum PS-CVT design was smaller than those of other multiple-speed layouts. Therefore, its application would be limited if greater performance constraints were imposed on the BEV. However, it should be noted that these results are limited to the case-study vehicle. Consequently, adoption of these transmission layouts for a BEV containing an EM with more diverse efficiency contours might result in more considerable reduction in energy consumption. Furthermore, using efficiency contours instead of constant values for calculation of efficiency of two- and three-speed transmissions might change the outcome.

\section{Nomenclature}

$\begin{array}{ll}\text { BEV } & \text { Battery Electric Vehicle } \\ \text { CVT } & \text { Continuously Variable Transmission } \\ \text { EM } & \text { Electric Motor } \\ \text { ESS } & \text { Energy Storage System } \\ \text { FR } & \text { Fixed-Ratio mechanism } \\ \text { FT - I } & \text { PS-CVT equipped with full-toroidal } \\ & \text { CVT and type I power flow } \\ \text { FT - III } & \text { PS-CVT equipped with full-toroidal } \\ & \text { CVT and type III power flow } \\ \text { HEV } & \text { Hybrid Electric Vehicle } \\ \text { ICE } & \text { Internal Combustion Engine } \\ \text { PB - I } & \text { PS-CVT equipped with push belt CVT } \\ \text { PB - III } & \text { and type I power flow } \\ & \text { and type III power flow } \\ \text { PG } & \text { Planetary Gear train } \\ \text { PS - CVT } & \text { Power-Split Continuously } \\ & \text { Variable Transmission } \\ \text { PSO } & \text { Particle Swarm Optimization } \\ \text { SOC } & \text { State Of Charge } \\ \text { UCDS } & \text { Unified Cycle Driving Schedule } \\ \text { UDDS } & \text { Urban Dynamometer Driving Schedule } \\ f_{0} & \text { First coefficient of coast-down test } \\ f_{1} & \text { Second coefficient of coast-down test } \\ f_{2} & \text { Third coefficient of coast-down test } \\ & \end{array}$

$\begin{array}{ll}f_{\text {res }} & \text { Resistance force } \\ f_{t} & \text { Traction force on tires } \\ F & \text { Objective function } \\ M & \text { Mass of vehicle } \\ P & \text { Power crossing each component } \\ \nu & \text { Speed of vehicle } \\ \eta & \text { Efficiency of elements } \\ \tau & \text { Speed ratio of powertrain } \\ \omega & \text { Rotational speed }\end{array}$

\section{References}

1. Mofijur, M., Rasul, M.G., Hyde, J., Azad, A.K., Mamat, R., and Bhuiya, M. M.K. "Role of biofuel and their binary (diesel-biodiesel) and ternary (ethanolbiodiesel-diesel) blends on internal combustion engines emission reduction", Renew. Sustainable Energy Rev., 53, pp. 265-278 (2016).

2. Karvonen, M., Kapoor, R., Uusitalo, A., and Ojanen, V. "Technology competition in the internal combustion engine waste heat recovery: a patent landscape analysis", J. Clean. Prod., 112(5), pp. 3735-3743 (2016).

3. Fotouhi, A., Auger, D.J., Propp, K., Longo, S., and Wild, M. "A review on electric vehicle battery modelling: From lithium-ion toward lithium-sulphur", Renew. Sustainable Energy Rev., 56, pp. 1008-1021 (2016).

4. Ren, Q., Crolla, D.A., and Morris, A. "Effect of transmission design on electric vehicle (EV) performance", Vehicle Power and Propulsion Conference, VPPC'09. IEEE, pp. 1260-1265 (2009).

5. Di Nicola, F., Sorniotti, A., Holdstock, T., Viotto, F., and Bertolotto, S. "Optimization of a multiple-speed transmission for downsizing the motor of a fully electric vehicle", SAE Int. J. Alt. Power., 1(1), pp. 134-143 (2012).

6. Walker, P., Rahman, S.A., Zhang, N., Zhan, W., Lin, Y., and Zhu, B. "Modelling and simulation of a two speed electric vehicle", Sustainable Automotive Technologies, Springer Berlin Heidelberg, pp. 193-198 (2012).

7. Gao, B., Liang, Q., Xiang, Y., Guo, L., and Chen, H. "Gear ratio optimization and shift control of 2-speed I-AMT in electric vehicle", Mech. Syst, Signal Pr., 5051, pp. 615-631 (2015).

8. Gao, B., Xiang, Y., Chen, H., Liang, Q., and Guo, L. "Optimal trajectory planning of motor torque and clutch slip speed for gear shift of a two-speed electric vehicle", J. Dyn. Syst. Meas. Control, 137(6), pp. 061016-061016 (2015).

9. Zhang, Z., Zuo, C., Hao, W., Zuo, Y., Zhao, X.L., and Zhang, M. "Three-speed transmission system for purely electric vehicles", Int. J. Auto. Tech., 14(5), pp. 773-778 (2013). 
10. Srivastava, N. and Haque, I. "A review on belt and chain continuously variable transmissions (CVT): Dynamics and control", Mech. Mach. Theory, 44(1), pp. 19-41 (2009).

11. Sorniotti, A., Subramanyan, S., Turner, A., Cavallino, C., Viotto, F., and Bertolotto, S. "Selection of the optimal gearbox layout for an electric vehicle", $S A E$ Int. J. Engines, 4(1), pp. 1267-1280 (2011).

12. Ruan, J., Zhang, N., and Walker, P. "Comparing of single reduction and CVT based transmissions on battery electric vehicle", Proceedings of the 14th IFToMM World Congress, Taiwan, pp. 610-618 (2015).

13. Mantriota, G. "Theoretical and experimental study of a power split continuously variable transmission system Part 1", P. I. Mech. Eng. D-J. Aut., 215(7), pp. $837-850$ (2001).

14. Mangialardi, L. and Mantriota, G. "Power flows and efficiency in infinitely variable transmissions", Mech. Mach. Theory, 34(7), pp. 973-994 (1999).

15. Bottiglione, F., De Pinto, S., Mantriota, G., and Sorniotti, A. "Energy consumption of a battery electric vehicle with infinitely variable transmission", Energies, 7(12), pp. 8317-8337 (2014).

16. Zhou, X., Walker, P., Zhang, N., Zhu, B., and Ding, F. "The influence of transmission ratios selection on electric vehicle motor performance", ASME Int. Mech. Eng. Congress Expo., Houston, Texas USA, pp. 289296 (2012).

17. Ruan, J., Zhang, N., and Walker, P. "Comparing of the effects of CVT and DCT on the EVs including braking energy recovery", 8th Australasian Cong. on Applied Mech., Melbourne, Australia, p. 942 (2014).

18. Zhu, B., Zhang, N., Walker, P., Zhou, X., Zhan, W., Wei, Y., and Ke, N. "Gear shift schedule design for multi-speed pure electric vehicles", P.I. Mech. Eng. DJ. Aut., 229(1), pp. 70-82 (2014).

19. Musio, C. and Damiano, A. "Analysis of the effects of gear transmission structure on the electric vehicle performances", International Conference on Electrical Systems for Aircraft, Railway, Ship Propulsion and Road Vehicles (ESARS), Aachen, Germany, pp. 1-6 (2015).

20. Walker, P.D., Roser, H., Zhang, N., and Fang, Y., Comparison of Powertrain System Configurations for Electric Passenger Vehicles, SAE Technical Paper (2015).

21. Zhang, L., Li, L., Qi, B., and Song, J., Configuration Analysis and Performance Comparison of Drive Systems for Pure Electric Vehicle, SAE Technical Papers (2015).

https://www.sae.org/publications/technical-papers/ content/2015-01-1165/
22. Ruan, J., Walker, P., and Zhang, N. "A comparative study energy consumption and costs of battery electric vehicle transmissions", Appl. Energy, 165, pp. 119-134 (2016).

23. Delkhosh, M., Foumani, M.S., and Boroushaki, M. "Geometrical optimization of parallel infinitely variable transmission to decrease vehicle fuel consumption", Mech. Based Des. Struc., 42(4), pp. 483-501 (2014).

24. Mantriota, G. "Performances of a parallel infinitely variable transmissions with a type II power flow", Mech. Mach. Theory, 37(6), pp. 555-578 (2002).

25. Mantriota, G. "Power split continuously variable transmission systems with high efficiency", P.I. Mech. Eng. D-J. Aut., 215(3), pp. 357-358 (2001).

26. AïtTaleb, A., Chaâba, A., and Sallaou, M. "Efficiency evaluation of continuously variable transmissions including a planetary gear train", Energy and Power Engineering, 5(2), p. 153 (2013).

27. Bottiglione, F. and Mantriota, G. "Effect of the ratio spread of CVU in automotive kinetic energy recovery systems", J. Mech. Design, 135(6), p. 061001 (2013).

28. Mantriota, G. "Theoretical and experimental study of a power split continuously variable transmission system Part 2", P.I. Mech. Eng. D-J. Aut., 215(7), pp. 851-864 (2001).

29. Bottiglione, F. and Mantriota, G. "MG-IVT: An infinitely variable transmission with optimal power flows", J. Mech. Design, 130(11), pp. 112603 (2008).

30. Pennestrı, E. and Valentini, P.P. "A review of formulas for the mechanical efficiency analysis of two degreesof-freedom epicyclic gear trains", J. Mech. Design, 125(3), pp. 602-608 (2003).

31. Delkhosh, M. and Foumani, M.S. "Multi-objective geometrical optimization of full toroidal CVT", Int. J. Automot. Technol., 14(5), pp. 707-715 (2013).

32. Tang, Q. and Eberhard, P. "Cooperative motion of swarm mobile robots based on particle swarm optimization and multibody system dynamics", Mech. Based Des. Struc., 39(2), pp. 179-193 (2011).

33. Trelea, I.C. "The particle swarm optimization algorithm: convergence analysis and parameter selection", Inform. Process. Lett., 85(6), pp. 317-325 (2003).

34. Hollowell, W. "Partnership for a new generation of vehicles", Transportation Research Circular, 453, Irvine, California, pp. 26-29 (1996).

35. Tong, H.Y. and Hung, W.T. "A framework for developing driving cycles with on-road driving data", Transport Reviews, 30(5), pp. 589-615 (2010).

\section{Biographies}

Tahmoores Farjam received his BSc degree in Mechanical Engineering from Sharif University of Technology in 2014. Presently, he is MSc student of Mechanical Engineering at Sharif University of Technology, 
Tehran, Iran. His fields of interest are vehicle dynamics, control strategies, power transmission systems, and optimization algorithms.

Mahmoud Saadat Foumani received his PhD degree in Mechanical Engineering from Sharif University of Technology, Tehran, Iran, in 2002. He was a faculty member at Semnan University from 2002 to 2006 and is now a faculty member of the Mechanical Engineering Department at Sharif University of Technology. He teaches courses in the Applied Design group at undergraduate and graduate levels. His teaching focuses on mechanical engineering design, vehicle dynamics, chassis design, and advanced mathematics.

Mojtaba Delkhosh received his BSc, MSc, and $\mathrm{PhD}$ degrees in Mechanical Engineering from Sharif University of Technology in 2009, 2011, and 2016, respectively. Presently, he is post-doctoral researcher of Applied Mechanical Engineering at Sharif University of Technology, Tehran, Iran. His fields of interest are vehicle dynamics, machine design, vibration analysis, power transmission systems, optimization algorithms, and hybrid electric vehicles. 